\title{
A hot air driven thermoacoustic-Stirling engine
}

M.E.H. Tijani

\author{
S. Spoelstra
}

December 2013

ECN-W--13-057 


\title{
A hot air driven thermoacoustic-Stirling engine
}

\author{
M.E.H. Tijani*, S. Spoelstra \\ Energy Research Centre of the Netherlands (ECN), PO Box 1, 1755 ZG Petten, The Netherlands
}

\section{A R T I C L E I N F O}

\section{Article history:}

Received 29 January 2013

Accepted 9 April 2013

Available online 9 May 2013

\section{Keywords:}

Thermoacoustics

Engine

Stirling

\begin{abstract}
A B S T R A C T
Significant energy savings can be obtained by implementing a thermally driven heat pump into industrial or domestic applications [1]. Such a thermally driven heat pump uses heat from a high-temperature source to drive the system which upgrades an abundantly available heat source (industrial waste heat, air, water, geothermal). A way to do this is by coupling a thermoacoustic engine with a thermoacoustic heat pump. The engine is driven by a burner and produces acoustic power and heat at the required temperature. The acoustic power is used to pump heat in the heat pump to the required temperature. This system is attractive since it uses a noble gas as working medium and has no moving mechanical parts or sliding seals. This paper deals with the first part of this system: the engine. In this study, hot air is used to simulate the flue gases originating from a gas burner. This is in contrast with a lot of other studies of thermoacoustic engines that use an electrical heater as heat source. Using hot air resembles to a larger extent the real world application. The engine produces about $300 \mathrm{~W}$ of acoustic power with a performance of $41 \%$ of the Carnot performance at a hot air temperature of $620{ }^{\circ} \mathrm{C}$.
\end{abstract}

(C) 2013 Elsevier Ltd. All rights reserved.

\section{Introduction}

Conventional engines generate work from heat by moving a piston or turning a turbine. Thermoacoustic engines in contrast have no mechanical moving parts or sliding seals involved in the power cycle which makes them very reliable. They have also the advantages of using only an environmentally friendly working medium (noble gases), simple implementation, and the use of common materials. The thermodynamic cycle used by a thermoacoustic traveling-wave engine is similar to the Stirling cycle. In 1979 , it was recognized that the time phasing between the pressure and velocity of the gas in the regenerator of a Stirling system is the same as in a traveling acoustic wave [2]. In an attempt to eliminate the moving mechanical parts in a conventional Stirling engine, Ceperley [2] built a traveling-wave thermoacoustic engine which consists simply of a regenerator sandwiched between two heat exchangers placed inside an air filled looped tube. However, the engine didn't function due to large viscous losses in the regenerator and to mass streaming in the tube. It is only in 1999 that the first efficient thermoacoustic-Stirling engine was built by Backhaus et al. [3]. This engine converts heat into acoustic power with a thermal efficiency of $30 \%$, corresponding to $41 \%$ of the Carnot efficiency without moving mechanical parts. In 2009, Tijani et al. developed a

\footnotetext{
* Corresponding author. Tel.: +31 (0) 2224564029.

E-mail address: tijani@ecn.nl (M.E.H. Tijani).
}

thermoacoustic-Stirling engine which achieved a record performance of $49 \%$ of the Carnot performance [4]. These achievements will pave the way for the thermoacoustic technology to industrial applications.

This paper presents the design, construction, and test of a hot air driven thermoacoustic heat engine. The engine uses heat $Q_{h}$ from hot air at a high temperature $T_{\mathrm{h}}$ to generate acoustic power $W_{\mathrm{a}}$. Part of the acoustic power is dissipated in the acoustic resonator (tube) and the rest (useful) is dissipated in a dummy variable acoustic load. The remaining heat $\left(Q_{a}\right)$ and the dissipated acoustic power $\left(Q_{r}\right.$ and $\left.Q_{l}\right)$ are rejected to the environment $\left(T_{\mathrm{a}}\right)$ as shown in Fig. 1 . The foreseeable industrial application requires that the engine achieves a performance of $40 \%$ of the Carnot performance. The work presented in this paper concerns the design, development, and test of the engine.

The remaining of this paper is organized as follow: Section 2 describes the working principle, design, and construction of the engine. Section 3 is devoted to the measurements procedure. Section 4 presents the experimental results. In Section 5 some conclusions are drawn.

\section{Design and construction of the thermoacoustic engine}

A schematic illustration of the thermoacoustic Stirling-engine is shown in Fig. 2. The thermoacoustic engine consists of a torusshaped section attached to a quarter-wavelength acoustic resonator and to a variable acoustic load. The system is filled with a 


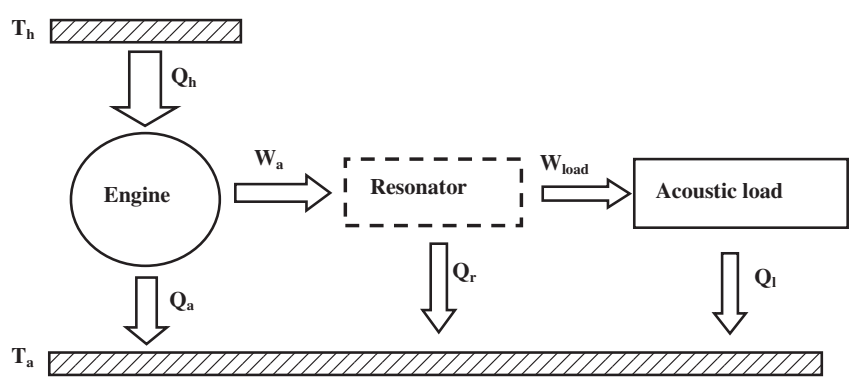

Fig. 1. Thermodynamic illustration of the thermoacoustic heat engine.

working gas (helium) at a given pressure. The torus-shaped section contains the thermodynamic active part consisting of a regenerator (REG.) sandwiched between two heat exchangers AHX1 and HHX. The regenerator is the heart of the engine where the thermoacoustic conversion processes take place. The thermoacousticStirling heat engine functions like an acoustic amplifier. The application of heat to the HHX while maintaining AHX1 at ambient temperature creates a temperature difference across the regenerator which generates spontaneously an acoustic wave $[2,3]$. The acoustic wave takes care of the compression, displacement, expansion, and the timing necessary for the Stirling cycle. To keep the process ongoing, part of the acoustic power is fed back through the feedback tube (L) to the ambient side (AHX1) of the regenerator to be amplified.

The remainder of the acoustic power is available as useful power at the junction to the resonator. The gas column in the thermal buffer tube (TBT) provides thermal insulation for HHX. A secondary ambient heat exchanger (AHX2) is placed at the end of the TBT to intercept the heat leaking down the TBT. A drawing of the torus part of the engine part is shown in Fig. 3.

The acoustic resonator is of a quarter wavelength type consisting of a straight cylindrical tube with a small diameter connected to a larger diameter cylindrical tube by means of a conical tube. The variable acoustic load, consisting of a tank and an adjustable valve, controls the power output of the engine.

The thermoacoustic computer code DeltaEC [5] is used to design and optimize the engine. The engine uses helium at $40 \mathrm{bar}$ and the operation frequency is $120 \mathrm{~Hz}$. Air, driven by a blower and heated by an electrical heater is used as heat source and water flow chilled by a thermostat bath carries away the heat rejected by the engine at AHX1. The different components of the engine as incorporated in the DeltaEC-model are indicated in Fig. 2. A summary of the optimized dimensions of the different parts of the engine is given in the following.

The regenerator consists of a $6 \mathrm{~cm}$ long stack of 180 -mesh stainless steel screens with a diameter of $6.7 \mathrm{~cm}$. The diameter of the screen wire is $40 \mu \mathrm{m}$. A volume porosity of $78 \%$ and a hydraulic radius of $35 \mu \mathrm{m}$ are calculated using the mesh-number and wire diameter.

To avoid structural and gas leakage problems which might appear due to the high temperature and high pressure; the regenerator holder, HHX, and TBT are made from a single high temperature steel block as shown in Fig. 4 (middle).

The first ambient heat exchanger (AHX1) is of cross-flow type and consists of a cylindrical brass block with a length in the acoustical direction of $2 \mathrm{~cm}$ and a diameter of $6.7 \mathrm{~cm}$ containing copper fins at the helium-side to increase the heat transfer area. The water-side consists of rectangular channels (slits) $1 \mathrm{~mm}$ high and $15 \mathrm{~mm}$ wide. A picture of AHX1 is shown in Fig. 4 (left).

The hot heat exchanger (HHX) is the most crucial component of the engine as it has to transfer heat between hot air and the working gas (helium). It is chosen to use a finfin cross-flow heat exchanger. The optimization of the heat exchanger consists of transferring the needed heat with minimal pressure drop. Slits are made in the block for both helium- and air-side using an EDM technique. However, because of the length of the slits and the difficulty to access them, this process took a long time and the cost was proportionally high. Stainless steel fins are brazed in the slits to improve heat transfer between helium and air. For the helium side 50 fins/inch are used with a length in the acoustical direction of $5 \mathrm{~cm}$ and a height of $1 \mathrm{~mm}$. For the air side 20 fins/inch are used with a cross-flow length of $7.5 \mathrm{~cm}$, a width in the acoustical direction of $4.5 \mathrm{~cm}$, and a height of $2 \mathrm{~mm}$.

The hot heat exchanger is designed to exchange $2 \mathrm{~kW}$ of heat between the hot air and helium gas. The length in the acoustic direction is determined by the peak-peak displacement of helium, which is $5 \mathrm{~cm}$; and the diameter of the heat exchanger has the same diameter as the regenerator, which is $6.7 \mathrm{~cm}$. The second ambient heat exchanger (AHX2) consists of a circular brass block containing 782 holes with a diameter of $1.5 \mathrm{~mm}$ and a length of $1 \mathrm{~cm}$ through which the helium gas oscillates. Water flows around the perimeter of this block to carry away the heat leaking down the TBT. A picture of AHX2 is shown in Fig. 4 (right). The thermal buffer tube consists of a thin-walled cylindrical tube with an inside diameter of $7 \mathrm{~cm}$, a wall thickness of $1.6 \mathrm{~mm}$, and a length of $17 \mathrm{~cm}$. It is made in the same block as the HHX as shown in Fig. 4. The feedback inertance consists of cylindrical tube with a length of $73 \mathrm{~cm}$ and diameter of $6.7 \mathrm{~cm}$. The pressure balanced sliding joint is used to compensate the thermal expansion of the hot parts of the engine. This will avoid the building of stress in the hot walls and hence the damage of these parts. The variable acoustic load consists of a $1 \mathrm{~L}$ tank and an adjustable valve. As indicated earlier, the resonator consists of two straight tubes connected by a cone. The first straight tube has an inner diameter of $8.3 \mathrm{~cm}$ and length of $86 \mathrm{~cm}$, the conical tube has a start inner diameter of $8.3 \mathrm{~cm}$, a length of $210 \mathrm{~cm}$, and a final inner diameter of $21.3 \mathrm{~cm}$. The last tube has an inner diameter of $21.3 \mathrm{~cm}$ and a length of $50 \mathrm{~cm}$. It is worthwhile to notice that an elastic membrane is placed at the ambient heat exchanger to suppress

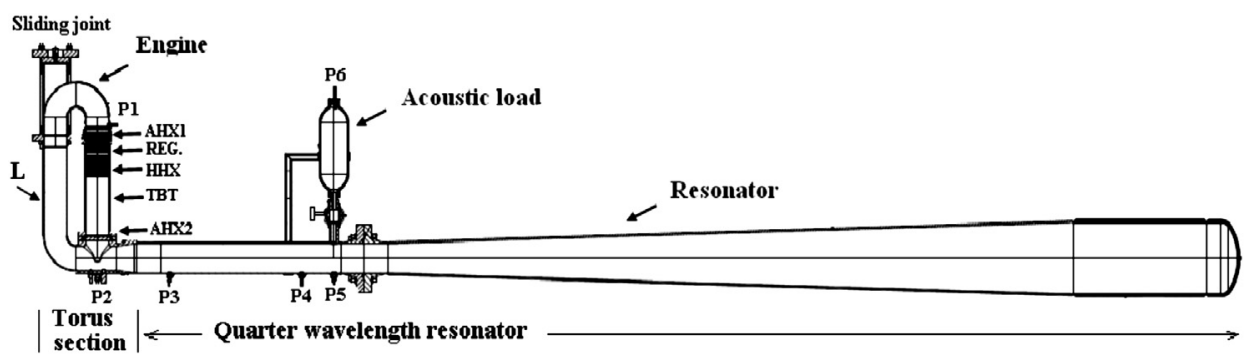

Fig. 2. Schematic illustration of the thermoacoustic Stirling-engine. An acoustic load is placed on the resonator to control the power output of the engine. The pressure sensors are indicated by "P's". 

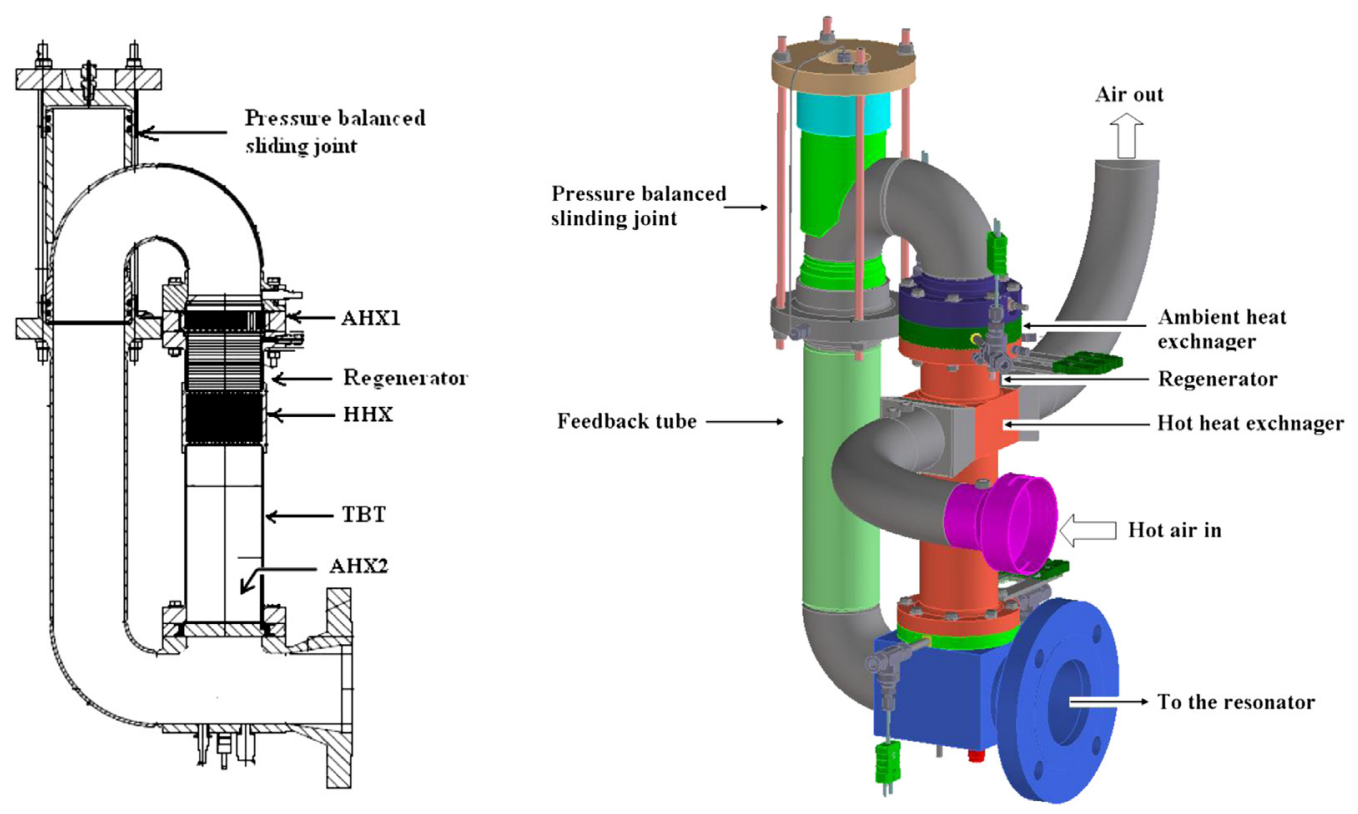

Fig. 3. A schematic- (left) and a CAD-drawing of the torus part of the engine.

Gedeon streaming [6] which is a second-order time-averaged mass flux which circulates around the torus removing heat from the hot heat exchanger and depositing it at AHX2 without taking part to the thermoacoustic conversion process in the regenerator. This causes a heat leak that seriously degrades the performance of the engine.

\section{Characterization of the engine}

The characterization of the performance of the engine requires the measurement of thermal and acoustic powers. The system is instrumented so that these different quantities can be measured. The thermal power input to the engine delivered by hot air flow or the thermal power extracted by flow water from the AHX1 or AHX2 is given by

$\dot{Q}=\rho c_{p} U\left(T_{0}-T_{\mathrm{i}}\right)$

Here is $\rho$ the density of hot air or water, $c_{p}$ is the specific heat of air or water, $U$ is the volume flow rate of air or water, and $T_{\mathrm{i}}$ and $T_{\mathrm{o}}$ are the input and output temperatures of the stream flowing through the heat exchanger. The volume flow rates of air and water are measured with flow meters and the temperatures are measured by K-type thermocouples placed at the inlet and outlet of the heat exchangers.

The acoustic power produced by the engine is measured by the so-called two-microphone method [3,7]. By reference to Fig. 2, the acoustic power flowing past the midpoint of the two pressure sensors $p_{3}$ and $p_{4}$ is given by $[3,7]$

$$
\dot{W}_{2 \text { mic }}=\frac{A}{2 \omega \Delta x \rho_{\mathrm{g}}}\left[\left(1-\frac{\delta_{\nu}}{r}\right) p_{3} p_{4} \sin \alpha+\frac{\delta_{\nu}}{2 r}\left(p_{3}^{2}-p_{4}^{2}\right)\right]
$$

Here $p_{3}$ and $p_{4}$ are the amplitudes of the dynamic pressures measured by the two pressure sensors, $\Delta x$ is the distance between the two transducers along the resonator, $\alpha$ is the phase angle by which $p_{3}$ leads $p_{4}, \omega$ is the angular frequency, $\rho_{\mathrm{g}}$ is the average density of the gas, and $\delta_{\nu}$ is the viscous penetration depth. The acoustic power dissipated in the load is given by Fusco et al. [7].

$\dot{W}_{\text {load }}=\frac{\omega V_{\mathrm{c}}}{2 \gamma p_{\mathrm{m}}} p_{5} p_{6} \sin \beta$

where $p_{5}$ and $p_{6}$ are the amplitudes of the dynamic pressures measured at the entrance of the load and in the compliance of the load respectively, $\beta$ is the phase difference between $p_{5}$ and $p_{6}, \omega$ is the angular frequency, $V_{\mathrm{c}}$ is the volume of the tank of the load, $p_{\mathrm{m}}$ is the average pressure of the gas, and $\gamma$ is the ratio of the isobaric to isochoric specific heats. The power, measured by the twomicrophone method is the sum of the acoustic power, dissipated in the resonator section to the left of the midpoint of the twomicrophones and the acoustic power, dissipated in the load
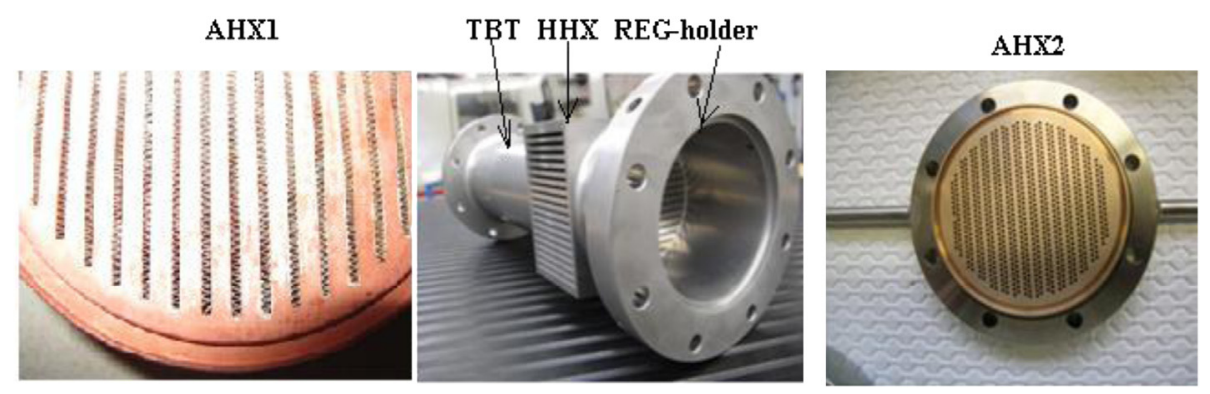

Fig. 4. (left) Picture of the AHX1; (middle) the block consisting of the regenerator holder, HHX, and TBT; (right) picture of AHX2. 

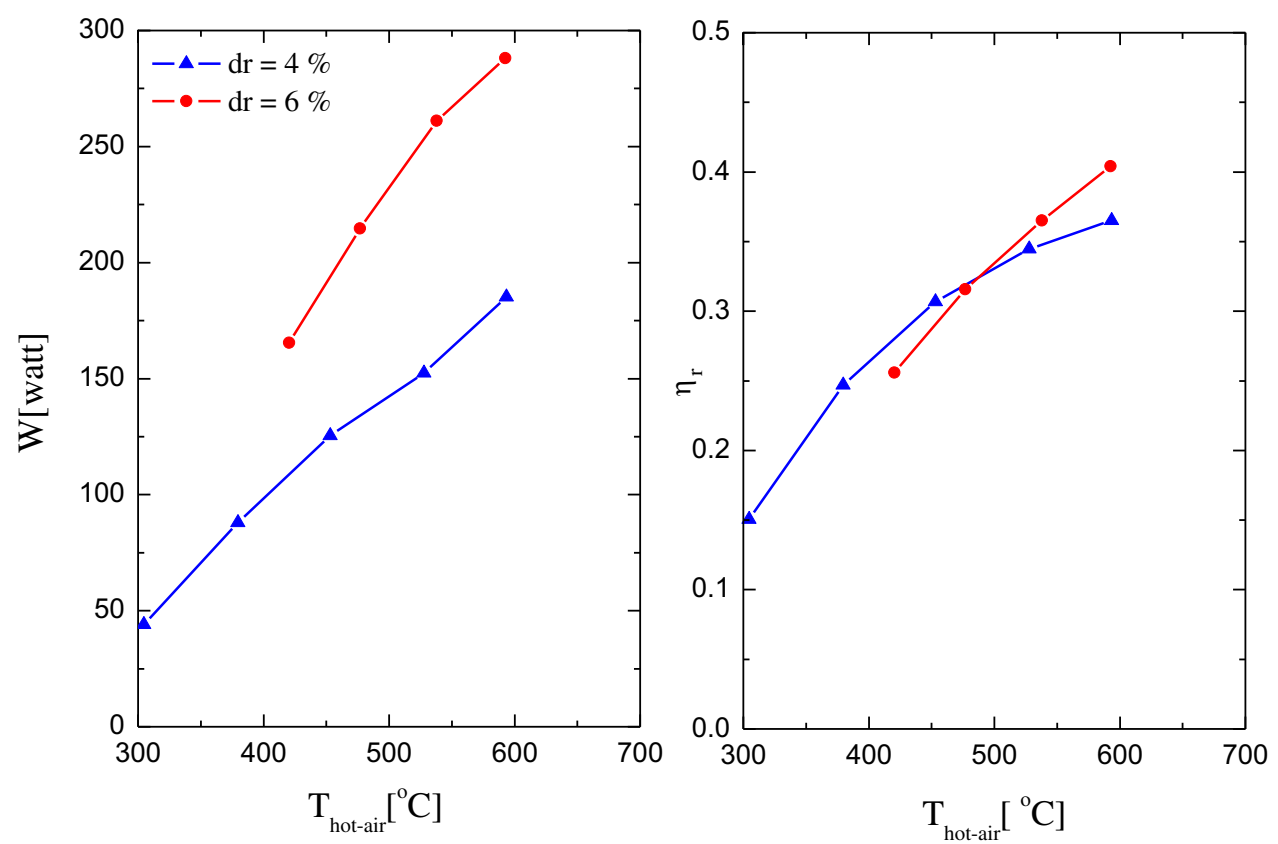

Fig. 5. Measured acoustic power and performance relative to Carnot as a function of the hot air temperature for two drive ratios 4 and $6 \%$.

$\dot{W}_{2 \text { mic }}=\dot{W}_{\text {res }}+\dot{W}_{\text {load }}$

It is worthwhile to note that the two-microphone method is quite difficult due to its sensitivity to the microphone position, the phase difference, and flow conditions. The performance of the engine $\eta$ is given by

$\eta=\frac{\dot{W}}{\dot{Q}_{\mathrm{h}}}$

where $\dot{W}$ is the acoustic power produced by the engine (entering the resonator) which is deduced from $\dot{W}_{2 \text { mic }}$ by extrapolation using the DeltaEC model of the system. The Carnot efficiency of the engine is given by

$\eta_{\mathrm{c}}=\frac{T_{\mathrm{h}}-T_{\mathrm{a}}}{T_{\mathrm{h}}}$

Here $T_{\mathrm{h}}$ is the temperature of the hot air and $T_{\mathrm{a}}$ is the average temperature of the cooling water flowing through the ambient heat exchanger. The performance relative to Carnot is defined as the ratio

$\eta_{\mathrm{r}}=\frac{\eta}{\eta_{\mathrm{c}}}$

\section{Experimental results}

The accuracy of the acoustic power measurements is first checked by plotting Expression 4 for several drive ratio's. The drive ratio is the ratio of the acoustic pressure and the average pressure. Linear fits to experimental results show a slope of about 1 which gives confidence in the two-microphone method measurements [3].

Fig. 5 shows the measured performance of the thermoacoustic engine as a function of the temperature of the hot air for two drive ratio's. The acoustic power output and the performance relative to Carnot increase as a function of the hot air temperature. At a given hot air temperature the acoustic power produced by the engine at a dive ratio of $6 \%$ is higher than at $4 \%$. The engine produces about $300 \mathrm{~W}$ of acoustic power with a performance of $41 \%$ of the Carnot performance at a drive ratio of $6 \%$ and a hot air temperature of $620^{\circ} \mathrm{C}$. This relatively good performance is obtained in spite of a considerable heat leakage due to streaming in the TBT and the not optimal operation of HHX. Although a membrane is used and flow straighteners are placed in the TBT, an appreciable quantity of heat is still leaking down the TBT, which is measured at the AHX2. The flow of the hot air through the HHX decreases with the temperature which indicates an increase of the pressure drop across the HHX. This limits the heat input to the engine. An improvement of the performance might be expected by suppressing the heat leak in the TBT and improving the heat transfer in the HHX.

\section{Conclusion}

A hot air driven thermoacoustic-Stirling engine is designed, built, and tested. The engine produces about $300 \mathrm{~W}$ of acoustic power with a performance of $41 \%$ of the Carnot performance at a hot air temperature of $620^{\circ} \mathrm{C}$. The performance of the engine can be improved by suppressing the heat leak down the thermal buffer tube and improving the HHX. A better solution might be to use a hot head instead of the HHX. The hot head with fins can be directly heated by the hot gases as usually done in conventional Stirlingengines.

\section{Acknowledgements}

This work has been partly funded by the European Commission within the EU-FP7 energy program: Grant Agreement no. 226415, Thematic Priority: FP7-ENERGY-2008-FET, "THermoacoustic Technology for Energy Applications”, Acronym: THATEA.

\section{References}

[1] S. Spoelstra, M.E.H. Tijani, Thermoacoustic heat pumps for energy savings, Presented at the Seminar "Boundary Crossing Acoustics" of the Acoustical Society of the Netherlands, 2005. http://www.ecn.nl/docs/library/report/2005/ rx05159.pdf. See also http://www.thatea.eu/. 
[2] P.H. Ceperley, A pistonless Stirling engine - the traveling wave heat engine, J. Acoust. Soc. Am. 66 (1979) 1508-1513.

[3] S. Backhaus, G.W. Swift, A thermoacoustic Stirling heat engine: detailed study, J. Acoust. Soc. Am. 107 (2000) 3148-3166.

[4] M.E.H. Tijani, S. Spoelstra, A high performance thermoacoustic engine, J. Appl. Phys. (2011) 110-116.
[5] W.C. Ward, G.W. Swift, J.P. Clark, Interactive analysis, design, and teaching for thermoacoustics using DeltaEC, J. Acoust. Soc. Am. 123 (2008) 3546.

[6] D. Gedeon, DC gas flows in Stirling and pulse tube cryocoolers, in: R.G. Ross (Ed.), Cryocoolers 9, Plenum, New York, 1997, p. 385.

[7] A.M. Fusco, W.C. Ward, G.W. Swift, Two-sensor power measurement in lossy ducts, J. Acoust. Soc. Am. 91 (1992) 2229-2235 


\section{ECN}

Westerduinweg 3

P.O. Box 1

1755 LE Petten

1755 LG Petten

The Netherlands

The Netherlands

$\mathrm{T}+31885154949$

$F+31885158338$

info@ecn.nl

www.ecn.nl 